\title{
Influence of nonlinear effects on the three-photon excitation of L-Tryptophan in water using phase-shaped pulses
}

\author{
Alexander Patas, ${ }^{1, *}$ Georg Achazi, ${ }^{1}$ Christopher Winta, ${ }^{1}$ and Albrecht Lindinger ${ }^{1}$ \\ ${ }^{1}$ Fachbereich Physik, Freie Universität Berlin, \\ Arnimallee 14, 14195 Berlin, Germany
}

compiled: June 19, 2014

\begin{abstract}
We show that nonlinear effects like self-phase modulation in a sample have to be considered for phase control of three-photon excitations. Furthermore we demonstrate the control of three photon excitation of L-Tryptophan in water using a pulse shaping setup. Simulations of the propagation of the laser pulses in the cuvette exhibit a good agreement to the experimental fluorescence scans at different laser intensities and show large discrepancies when neglecting nonlinear effects prior to the three-photon process. This can lead to improvements in selective excitation of amino acids by a near infrared femtosecond laser source.
\end{abstract}

OCIS codes: $\quad 190.7110,300.2530,300.6410,320.5540$

http://dx.doi.org/10.1364/XX.99.099999

\section{Introduction}

Multiphoton microscopy has become a valuable tool for modern day research in biology and medicine. Since the developement of two-photon laser scanning microscopy [1], many publications have shown how the resolution can be enhanced $[2,3]$ or selective excitation of different fluorescent markers can be achieved [4-7].

In most applications of multiphoton microscopy infrared light is used for excitation, because it takes advantage of the favourable transmission window in biological tissue. Therefore only the fluorescence light has to travel back from the focus to be collected by a spectrometer or photomultiplier. This largely enhanced the achievable penetration depth when using this method of excitation. Recently three-dimensional images of a live mouse brain in vivo have been captured in a depth of $1600 \mu \mathrm{m}$ [8]. Later this was even achieved non-invasively [9] pointing out the usefullness of three-photon microscopy in $3 \mathrm{~d}-$ imaging. Many suitable marker substances have been identified and are now widely used [10], but utilizing the UV autofluorescence of cells for multiphoton microscopy could make the use of marker molecules redundant. However amino acids can be directly excited in a three-photon process using infrared light. This could be beneficial since amino acids as the building blocks of life are present in most biological samples. Tryptophan, which exhibits the most efficient fluorescence, is a well studied system. Transient absorption measurements, as well as excited state dynamics have been under investigation $[11,12]$. In different experiments, Chen et.al.

\footnotetext{
* Corresponding author: patas@physik.fu-berlin.de
}

[13] found optimal pulse shapes when optimizing threephoton over two-photon fluorescence while adjusting the parameter of an engineered optimization cost function. These results can be applied to three-photon microscopy which becomes more and more relevant as it allows for even better resolution and depth sensitivity.

Three-photon excitation in microscopy requires laser intensities which imply further nonlinear effects. Although these can be minimized in the optical setup by choosing a large beam diameter, spectral changes due to e.g. self-phase modulation (SPM) in the sample will be inevitable. Therefore we want to understand what changes have to be expected when applying phase shaping to three-photon excitation of L-Tryptophan in water and how pulse shapes can be used in combination with nonlinear effects to maximize possible selective excitation of biomolecules.

\section{Fundamental principles}

Nonresonant three-photon excitation relies just like nonresonant two-photon excitation on constructive interference of all possible excitation pathways leading to one excitation energy. The efficiency at which nonresonant excitation is possible can be characterized by the multiphoton spectrum of a laser pulse (Eq. 1) where $|E(\omega)|$ is the spectral electrical field envelope and $\Phi(\omega)$ is the corresponding spectral phase.

$$
\begin{aligned}
E^{(3)}(3 \omega)= & \int_{-\infty}^{\infty} \int_{-\infty}^{\infty}\left|E\left(\omega-\Omega_{1}-\Omega_{2}\right)\right|\left|E\left(\Omega_{1}\right)\right|\left|E\left(\Omega_{2}\right)\right| \\
& \times e^{i\left(\Phi\left(\omega-\Omega_{1}-\Omega_{2}\right)+\Phi\left(\Omega_{1}\right)+\Phi\left(\Omega_{2}\right)\right)} d \Omega_{1} d \Omega_{2} .
\end{aligned}
$$

This equation integrates over all possible photon 
triples with the total energy of $3 \hbar \omega$. Different from the case of the two-photon spectra where antisymmetric phase functions lead to full constructive intereference at the point of symmetry, only the constant phase function achieves maximal values in the case of three-photon excitation [14].

Simulations show that despite the complexity of the three-photonic case, antisymmetric phase functions can be used to shift positive interference in the three-photon spectrum to smaller or larger wavelengths. This could be utilized to selectively excite fluorphores with a changing absorption in the range of the laser spectrum. If the three-photon absorption cross-section $\left(A b s_{3 p}\right)$ is known one can then calculate the resulting fluorescence of the fluorophore by

$$
I \propto \int_{-\infty}^{\infty} E^{(3)}(3 \omega) A b s_{3 p}(3 \omega) d \omega .
$$

Because data of the three-photon absorption is not available, in our case we approximate this in the following simulations by the one-photon absorption crosssection. We think this is feasible, since regarding selection rules, every one-photon transition could as well be excited by three-photons.

\section{Experimental setup}

The laser system used for the measurements presented in this publication consists of an oscillator (Mira) and a regenerative amplifier (Rega 9000) which can reach pulse energies of up to $3 \mu \mathrm{J}$ at a rate of $287 \mathrm{kHz}$. The central wavelength was chosen to be $805 \mathrm{~nm}$ with a spectral bandwidth of $22 \mathrm{~nm}$.

The pulse modulation is achieved by a standard 4f setup and a spatial light modulator (SLM-640) from CRI. The resolution was measured to be $0.2716 \mathrm{~nm} / \mathrm{px}$. Just behind the pulse-shaping setup a polarizer was placed to allow for amplitude and phase modulation of linearly polarized pulses.

The pulse duration of about $58 \mathrm{fs}$ and the resulting peak intensities would lead to undesired white-light generation in the glass of the cuvette. Therefore additional neutral density filters as well as transmission modulation by the SLM were used to adjust the pulse energy in the course of the experiments.

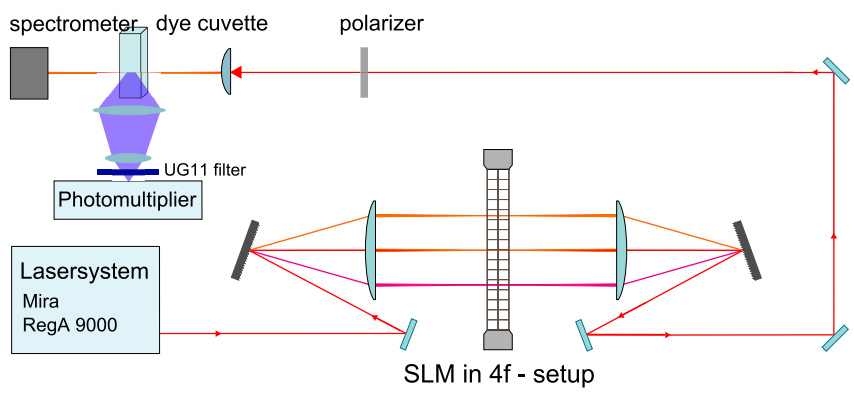

Fig. 1. Experimental setup
The laser pulses of various energy and phase were then focused in a $12 \mathrm{~mm}$ long cuvette filled with $15 \mathrm{mM} \mathrm{L-}$ Tryptophan in v.e. water (see fig 1 ). A lens with a focal length of $20 \mathrm{~cm}$ was placed in front of the fluorescence setup so that the focus was at the center of the cuvette at a depth of $6 \mathrm{~mm}$. The emitted light was then detected by two collecting lenses and a photomultiplier tube covered by an UG11 and an IR filter orthogonal to the incident light to minimize stray light.

In order to check if the parameters used in the simulation are correct a fiber spectrometer was placed behind the cuvette monitoring nonlinear changes to the pulse spectrum. In all measurements an offset phase was used to ensure that the reference pulse was transform-limited. This offset was found at low laser intensities by optimizing the fluorescence of Rhodamine B using the PRISM algorithm [15].

Since phase shaping can lead to undesired side effects like space time coupling we optimized the phase a second time using a two-photon absorbing diode placed in the non focused laser beam. The difference between the fluorescence and diode optimization seemed mainly due to the additional lens and dispersion of the cuvette. Therefore we concluded that the offset phase, as desired, only optimizes the pulse duration and not the spatial focus.

\section{Simulation and experimental results}

An implementation of the well-known split-step method [16] was used to determine the changes of the pulse shape and spectrum during the propagtion through the cuvette. Since nonlinear effects are very sensitive on the actual pulse intensity, the beam waist around the focus was taken into consideration as well. A Gaussian beam focus was used to evaluate the fluence at each point in the cuvette at which the simulation was then carried out. The nonlinear propagation considered the dispersion of quartz (cuvette wall) and water (sample), SPM and self-steepening. The group-velocity dispersion (GVD), third-order dispersion (TOD), and nonlinear refractive index $\left(n_{2}\right)$ values were taken from the literature [1720]. After the nonlinear propagation, the three-photon spectrum was calculated and the result was multiplied by the wavelength dependent one-photon absorption of Tryptophan.

As addition to the offset phase, precompensating the material dispersion, we used a third order phase function as parametrization for the shaped pulses.

$$
\Phi(\lambda)=b_{3} \frac{2 \pi}{6}\left(\frac{c}{\lambda}-\frac{c}{\lambda_{0}}\right)^{3} .
$$

Here, $\mathrm{c}$ is the speed of light, $\lambda_{0}$ the wavelength around which the phase is antisymmetric and $b_{3}$ the amplitude, which was selected to be $\pm 5 \times 10^{4} \mathrm{fs}^{3}$.

Since the nonlinear propagation of the laser pulses in the quartz and water of the cuvette is highly dependent on the product of the nonlinear refractive index and the laser intensity $\left(n_{2} \times I\right)$, we had to make sure all parameters used in the simulation were correct. Possible 


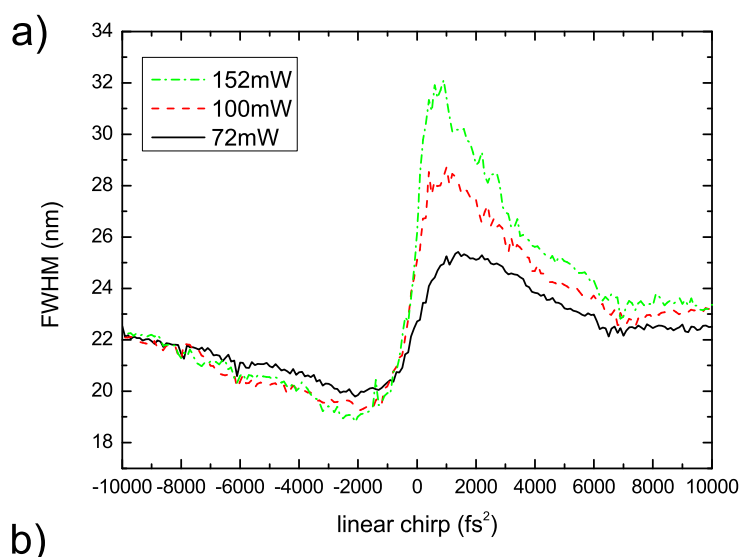

b)

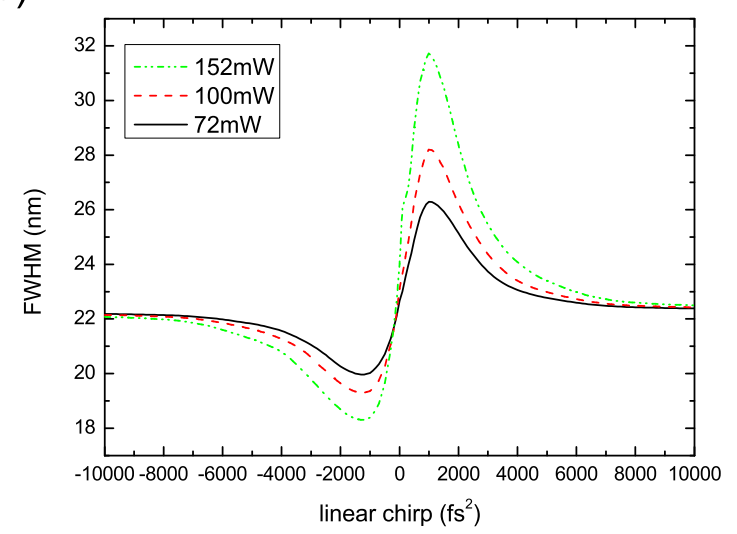

Fig. 2. a) Measurement of the spectral FWHM after the cuvette plotted against the linear pulse chirp at the focus position. b) Simulation of the propagated spectra for pulses of various linear chirps. A comparison is made at three different values for the laser power.

deviations in the average laser power measured by a powermeter and in the beam diameter may account for large uncertainties. Using the pulse shaper we varied the linear chirp (which equates to a quadratic phase function) of the precompensated laser pulses and measured each, mainly by SPM distorted, spectrum after the cuvette. The FWHM of these spectra were then compared to the simulation (see fig. 2).

The combination of just literature values with the pulse energy measured by the powermeter led to a surprisingly good agreement in the maximal and minimal FWHM of both simulation and measurement. This indicates that we can use this model to predict the influence of SPM and self-steepening on the three-photon excitation of Tryptophan.

Fig. 3 shows the simulated three-photon spectrum in the case of a third-order phase function of $\pm 5 \times 10^{4} \mathrm{fs}^{3}$ which is antisymmetric around $805 \mathrm{~nm}$ while neglecting nonlinear effects in the cuvette. As one can see, inverting the sign of the phase function has under these circumstances no effect on the three-photon spectra.

In contrast a calculation using the nonlinear Schrödinger equation (NLSE) [21] to estimate the
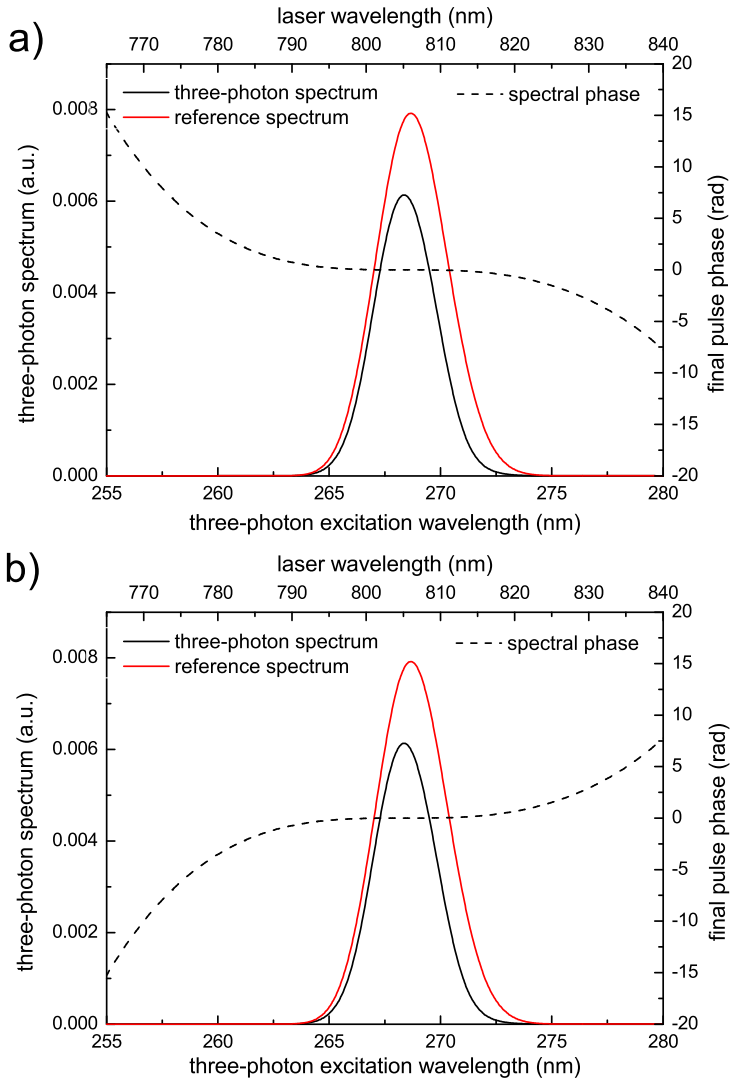

Fig. 3. a) third-order phase of $5 \times 10^{4} \mathrm{fs}^{3}$ and the resulting three-photon spectrum. b) third-order phase of $-5 \times 10^{4} \mathrm{fs}^{3}$. Without the effects of SPM and self-steepening both phase functions centered around $805 \mathrm{~nm}$ result in the same three-photon spectrum. The red line depicts the threephoton spectrum of the transform-limited pulse.

changes on the spectrum due to nonlinear effects leads to significant differences for both shaped pulses (see fig. 4). For each pulse-phase the propagation of the shaped laser pulse was simulated and the resulting spectra at the focus were used to evaluate the three-photon spectrum as in Eq.1 and 2. As a reference the dotted line in the background represents the three-photon spectrum of the transform-limited pulse after the pulse shaper.

The position of the phase's point of antisymmetry further defines the shape of the simulated three-photon spectrum. When shifting the point of antisymmetry to different wavelengths the maximum of the three-photon spectra shifts accordingly. In the case of a two-photon process it can still be analytically understood that antisymmetric phase functions reach full constructive interference at the wavelength of symmetry [22]. A rule of thumb for the case of a three-photon transition would be that antisymmetric phase functions still exhibit a defined maximum in the three-photon spectrum but unlike in the two-photonic case cannot reach the absolute constructive interference. The chosen function would then in addition need to be shallow around the wavelength 
a)

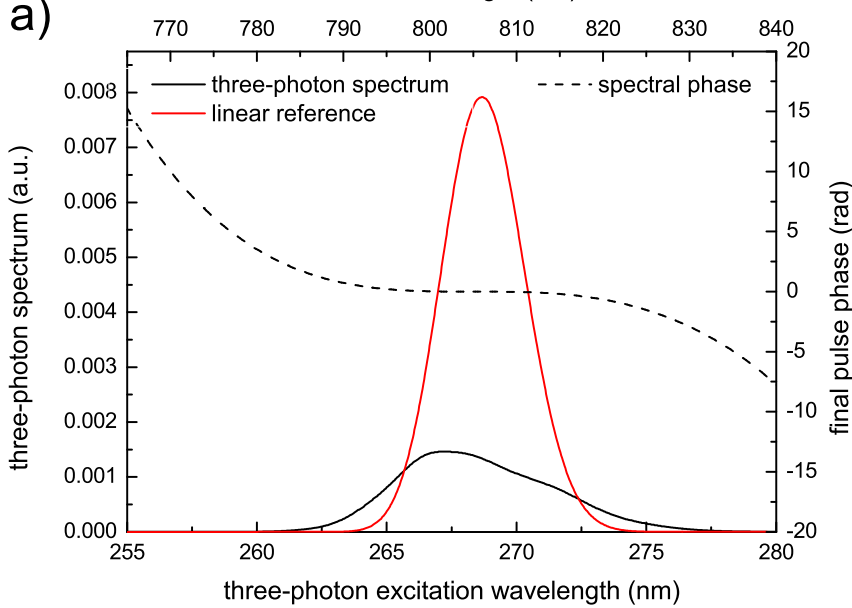

c)

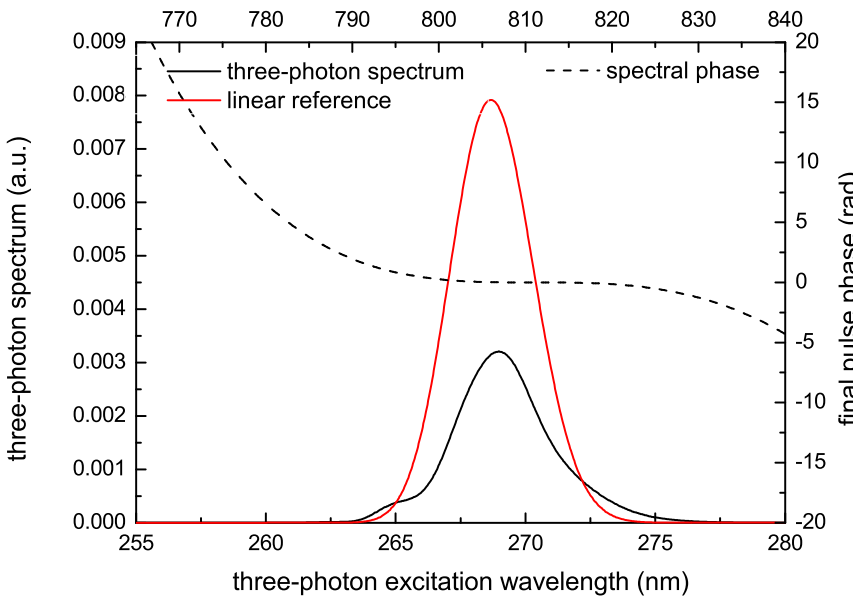

b)

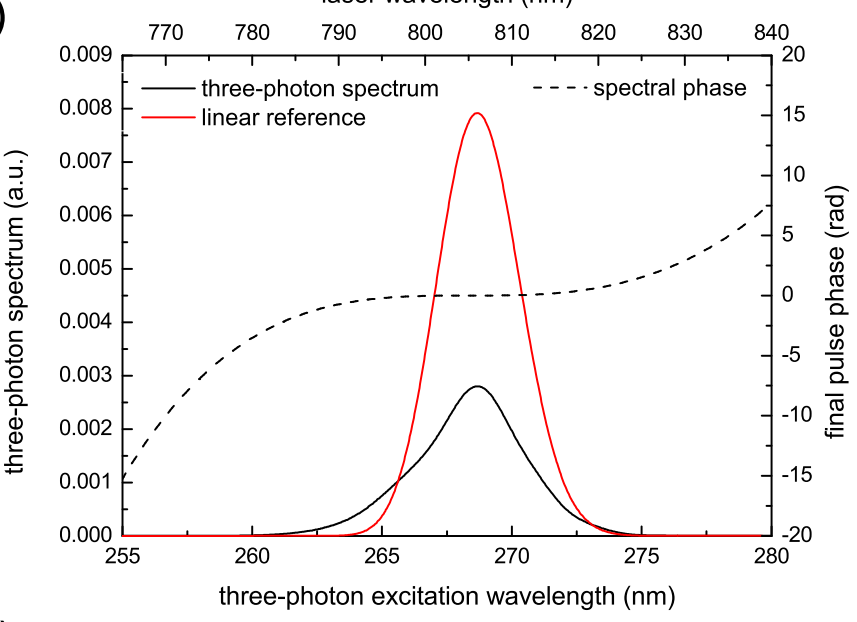

d)

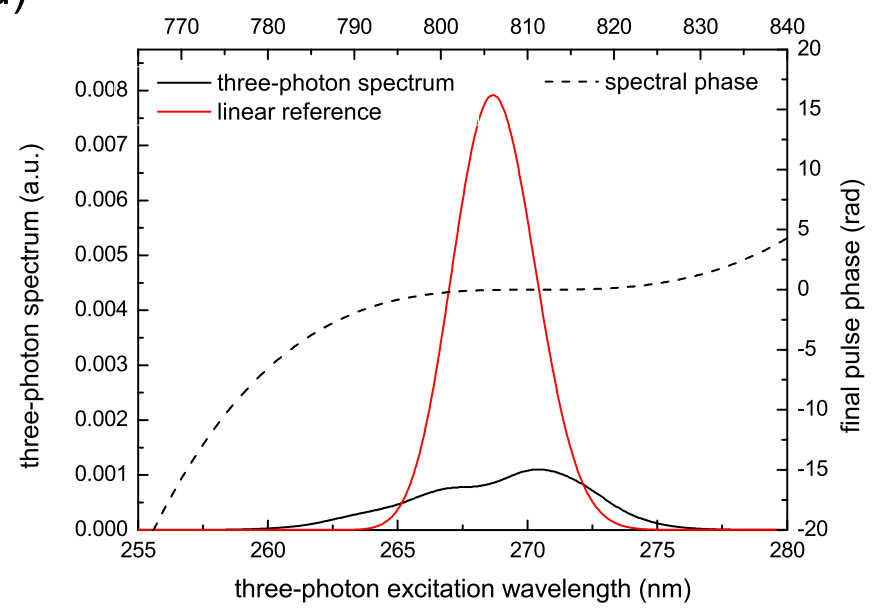

Fig. 4. Simulated three-photon spectra: a) $+5 \times 10^{4} \mathrm{fs}^{3}$, center wavelength $\left.805 \mathrm{~nm}, \mathrm{~b}\right)-5 \times 10^{4} \mathrm{fs}^{3}$, center wavelength $805 \mathrm{~nm}$, c) $+5 \times 10^{4} \mathrm{fs}^{3}$, center wavelength $811 \mathrm{~nm}$ and d) $-5 \times 10^{4} \mathrm{fs}^{3}$, center wavelength $811 \mathrm{~nm}$. When considering nonliear effects large differences in the three-photon spectra emerge.

at which we want to achieve three-photon excitation, thereby preventing the pulse's peak intensity to drop to low for efficient three-photon excitation.

The dominant nonlinear effect on the spectrum is clearly SPM while small changes were observed as well when self-steepening was disabled in the calculation. Because all pulses are modulated with a phase that is precompensating the dispersion of the cuvette up to the focus, we are in the regime of spectral narrowing until the focus is reached. On the way out of the cuvette we would expect mainly spectral broadening since the frequency shift induced by SPM is [21]

$$
\delta \omega(t)=-n_{2} \omega_{0} \frac{L}{c} \frac{\partial}{\partial t} I(t)
$$

Here $\mathrm{L}$ is the material length over which the effects of linear dispersion can be assumed to be small and $\mathrm{I}(\mathrm{t})$ the temporal intensity profile of the laser pulse. While SPM cannot lead to an overall frequency shift by itself

$$
\int_{-\infty}^{\infty} \frac{\partial I(t)}{\partial t} d t=[I(t)]_{-\infty}^{\infty}=0
$$

asymmetric pulse shapes can lead to asymmetric effects of SPM nonetheless. In the focus, where the laser fluence reaches values of $76 \mathrm{~mJ} / \mathrm{cm}^{2}$, the pulse that is shaped with a third-order phase has a steep leading flank and shallow trail or vice versa. In combination with the varying instantaneous frequency within the pulse a rather complex spectral change arises. Since this is unique for each shifted spectral phase, simulations will give the best insight into the expected spectral changes.

This effect can be observed when looking at the simulated spectra in the focus of the cuvette. The SPM and dispersion of $1 \mathrm{~mm}$ quartz and $5 \mathrm{~mm}$ water were therefore calculated using the pulse spectrum after the pulse shaper as a reference (see fig. 5). One clearly sees the shifted spectra depending on the sign of the cubic phase function. 


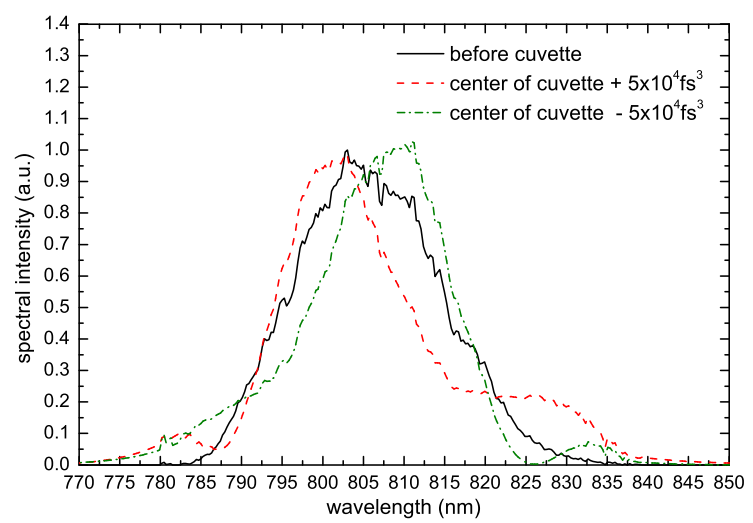

Fig. 5. This figure shows the simulated spectra for thirdorder phases of $\pm 5 \times 10^{4} \mathrm{fs}^{3}$ centered around $805 \mathrm{~nm}$ as well as the reference spectrum measured before the cuvette.

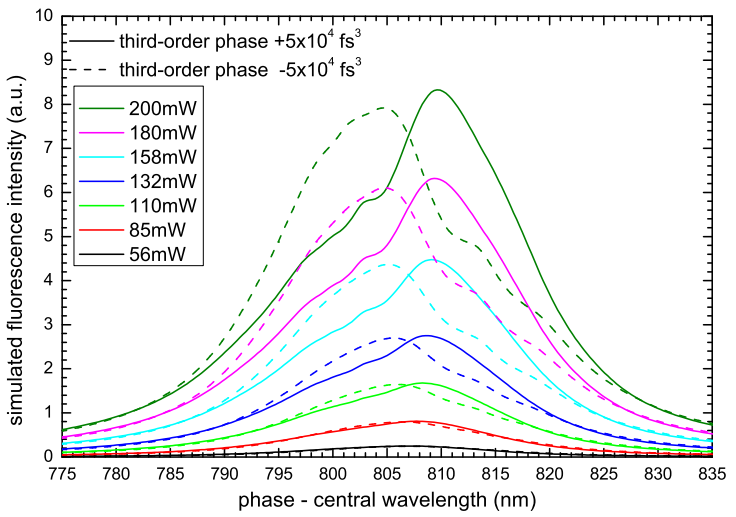

Fig. 6. The simulation of the expected fluorescence intensity of Tryptophan from pulses with a shifted $\pm 5 \times 10^{4} \mathrm{fs}^{3}$ thirdorder phase. First the nonlinear propagation of the pulses in the cuvette and then the three-photon process is simulated. This is done for each pulse shape at each laser power.

Fig. 6 shows the simulated fluorescence intensity for various phase shifts and intensities. The wavelength on the $\mathrm{x}$-axis depicts the center wavelength of the thirdorder phase function (see eq. 3). Both nonlinear effects, dispersion and efficiency of the three-photon process in addition to the one-photon absorption of Tryptophan are used to simulate the expected fluorescence of the amino acid. This approximation is plausible because one and three-photon processes feature the same symmetry. All simulation parameters were taken either from the literature (dispersion and nonlinear refractive index) or measured in preparation to the experiment. The spectral shift due to SPM is the main reason for the difference of fluorescence intensity when changing the center wavelength of the phase function. The seperation of the maxima increases for larger laser intensities and can be traced back to nonlinearities in the cuvette. This is confirmed by the vanishing seperation of both curves at low laser powers.

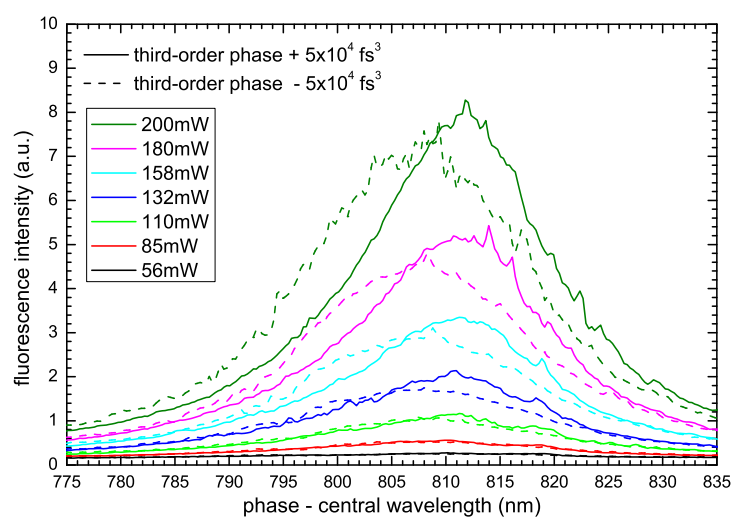

Fig. 7. Measured shifted-phase scans of a $\pm 5 \times 10^{4} \mathrm{fs}^{3}$ thirdorder phase for various laser powers. For low peak intensities in the cuvette nonlinear effects disappear while in the case of large fluorescence a shift between positiv and negative phase functions arises.

The experiment was done as described before: A third order phase function was applied to the pulse using the pulse shaper. Then the center wavelength of the phase function was shifted over the entire spectrum, thereby exciting the sample more efficiently at lower or higher wavelengths. Finally the fluorescence light was collected. The shifted-phase scans of the cubic phase resemble the simulation quite well (see fig. 7). The separation of the fluorescence maxima is increasing for larger laser power and nearly vanishes for values below $85 \mathrm{~mW}$. As observed in the simulation as well the intensity maxima of negatively shaped pulses are shifted to higher wavelengths while positively shaped pulses show the fluorescence maximum for phases antisymmetric around lower wavelengths.

\section{Conclusion and outlook}

Because of the good agreement of simulation and experiment, which was achieved without fitting simulation parameters, we conclude that these nonlinear effects can be estimated in good accuracy. Nonlinear effects seem to be very relevant in the regime of three-photon excitation, especially when pulse shaping is applied since different pulse shapes lead to varying nonlinear distortion of the exciting spectra. While the focal length of the lens in front of the cuvette might differ depending on various applications, the peak-intensities in the focus will be similar to the experiments presented in this work if a comparable fluorescence yield is to be reached. Since both, three-photon excitation and self-phase modulation are scaling with the intensity to the power of three both effects will occur in the focal area. Especially when strong fluorescence is required for spectroscopy, three-photon excitation is inherently coupled to spectral changes of the exciting laser spectrum in the sample. As shown in earlier publications [23, 24] one can achieve selective excitation of fluorophores by pulse shaping. This method could be applied as well to nonresonant three-photon 
processes. Using phase scans as shown in this paper or optimization algorithms it should be possible to achieve selective excitation exploiting nonlinear effects for further contrast enhancements. Three-photon microscopy has an even better depth sensivity than two-photon microscopy and can be used to excite biomolecules which absorb mainly in the near UV while keeping the excitation wavelength in the transmission window of tissue. It appeares that nonlinear effects in this type of application are well predictable and a compensation or calibration should be taken into account when using three-photon fluorescence microscopy.

We thank Prof. Dr. Ludger Wöste for his support.

\section{References}

[1] W. Denk, J. H. Strickler, and W. W. Webb. "Twophoton laser scanning fluorescence microscopy," Science 248, 73-76 (1990).

[2] S. Hell and E. H.K. Stelzer, "Fundamental improvement of resolution with a $4 \mathrm{Pi}$-confocal fluorescence microscope using two-photon excitation," Optics Communications 93, 277-282 (1992).

[3] R. Heintzmann and G. Ficz, "Breaking the resolution limit in light microscopy," Briefings in Functional Genomics \& Proteomics 5, 289-301 (2006).

[4] I. Pastirk, J. D. Cruz, K. Walowicz, V. Lozovoy, and M. Dantus, "Selective two-photon microscopy with shaped femtosecond pulses," Optics Express 11, 1695-1701 (2003).

[5] J. P. Ogilvie, D. Débarre, X. Solinas, J. Martin, E. Beaurepaire, and M. Joffre, "Use of coherent control for selective two-photon fluorescence microscopy in live organisms," Optics Express 14, 759-766 (2006).

[6] J. D. Cruz, V. Lozovoy, and M. Dantus, "Coherent control improves biomedical imaging with ultrashort shaped pulses," Journal of Photochemistry and Photobiology A: Chemistry 180 307-313, (20069.

[7] R. Pillai, C. Boudoux, G. Labroille, N. Olivier, I. Veilleux, E. Farge, M. Joffre, and E. Beaurepaire, "Multiplexed two-photon microscopy of dynamic biological samples with shaped broadband pulses," Optics Express 17, 12741-12752 (2009).

[8] D. Kobat, N. Horton, and C. Xu, "In vivo two-photon microscopy to 1.6-mm depth in mouse cortex," Journal of Biomedical Optics 16, 106014 (2011).

[9] N. Horton, K. Wang, D. Kobat, C. Clark, F. Wise, C. Schaffer, and C. Xu, "In vivo three-photon microscopy of subcortical structures within an intact mouse brain," Nature photonics 7, 205-209 (2013).

[10] C. Xu, W. Zipfel, J. B. Shear, R. Williams, and W. Webb, "Multiphoton fluorescence excitation: new spectral windows for biological nonlinear microscopy," Pro- ceedings of the National Academy of Sciences 93, 1076310768 (1996).

[11] S. Schenkl, F. van Mourik, N. Friedman, M. Sheves, R. Schlesinger, S. Haacke, and M. Chergui, "Insights into excited-state and isomerization dynamics of bacteriorhodopsin from ultrafast transient UV absorption," Proceedings of the National Academy of Sciences of the United States of America 103, 4101-4106 (2006)

[12] D. Sharma, J. Léonard and S. Haacke, "Ultrafast excited-state dynamics of tryptophan in water observed by transient absorption spectroscopy ," Chemical Physics Letters 489, 99-102 (2010)

[13] J. Chen, H. Kawano, Y. Nabekawa, H. Mizuno, A. Miyawaki, T. Tanabe, F. Kannari, and K. Midorikawa, "Selective excitation between two-photon and threephoton fluorescence with engineered cost functions," Optics Express 12, 3408-3414 (2004).

[14] V. Lozovoy, I. Pastirk, K. Walowicz, and M. Dantus, "Multiphoton intrapulse interference. ii. control of twoand three-photon laser induced fluorescence with shaped pulses," The Journal of Chemical Physics 118, 31873196 (2003).

[15] T. Wu, J. Tang, B. Hajj, and M. Cui, "Phase resolved interferometric spectral modulation (prism) for ultrafast pulse measurement and compression," Opt. Express 19, 12961-12968 (2011).

[16] G. Agrawal, Nonlinear fiber optics (Springer, 2000).

[17] Newport, "The effect of dispersion on ultrashort pulses," http://www.newport.com/the-effect-of-dispersion-onultrashort-pulses/602091/1033/content.aspx ,(2014).

[18] A. Couairon, L. Sudrie, M. Franco, B. Prade, and A. Mysyrowicz, "Filamentation and damage in fused silica induced by tightly focused femtosecond laser pulses," Phys. Rev. B 71, 125435 (2005).

[19] P. Devi, V. Lozovoy, and M. Dantus, "Measurement of group velocity dispersion of solvents using 2-cycle femtosecond pulses: Experiment and theory," AIP Advances 1, 032166 (2011).

[20] Z. Wilkes, S. Varma, Y. Chen, H. Milchberg, T. Jones, and A. Ting, "Direct measurements of the nonlinear index of refraction of water at 815 and $407 \mathrm{~nm}$ using singleshot supercontinuum spectral interferometry," Applied Physics Letters 94, 211102 (2009).

[21] R. Boyd, Nonlinear optics (Academic press, 2003).

[22] D. Meshulach and Y. Silberberg, "Coherent quantum control of multiphoton transitions by shaped ultrashort optical pulses," Phys. Rev. A 60, 1287-1292 (1999).

[23] A. Patas, G. Achazi, N. Hermes, M. Pawłowska, and A. Lindinger, "Contrast optimization of two-photon processes after a microstructured hollow-core fiber demonstrated for dye molecules," Applied Physics B 112, 579586 (2013).

[24] G. Achazi, N. Hermes, A. Patas, D. Tolksdorf, and A. Lindinger, "Polarization-shaped laser pulses for improved fluorescence anisotropy contrast," The European Physical Journal D 67, 1-5 (2013). 